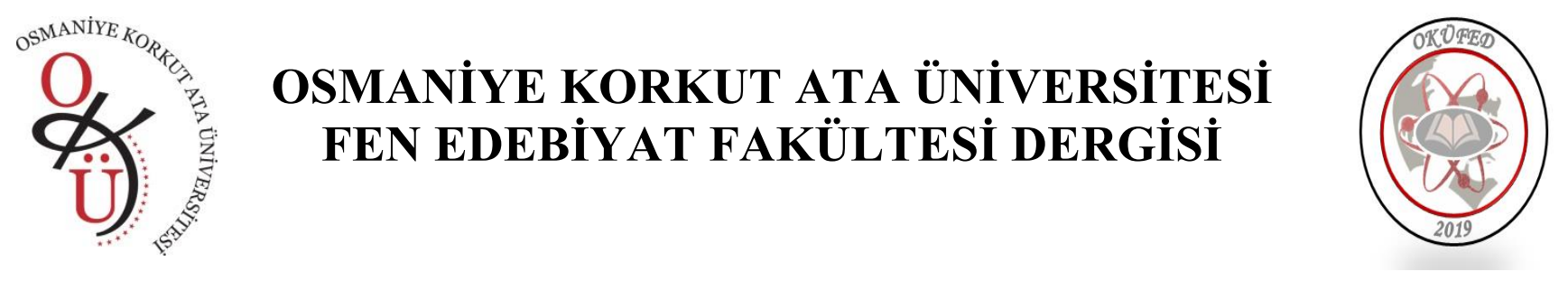

\title{
A Study on the Solution of the Extrapolation Distance Problem Using $U_{1}$ Method
}

\author{
Cevat AKSU ${ }^{1}$, Hakan ÖZTÜRK ${ }^{2}$
}

Received/ 27.12.2021

Published/17.01.2022

\begin{abstract}
The neutron transport equation for one-speed neutrons in a homogeneous slab was solved for the solution of the extrapolation distance problem. In the solution algorithm of the problem first the traditional $P_{1}$ method in which the neutron angular flux was expanded in terms of the Legendre polynomials and then the $U_{1}$ method in which the neutron angular flux was expanded in terms of the Chebyshev polynomials of second kind were used. While it is possible to find the solution of the problem obtained by $P_{1}$ method in literature, $U_{1}$ method was first applied to this problem and an analytical expression was obtained as the main goal of this study. Finally, numerical results for the extrapolation distances were calculated using both methods and they were given in the tables together with the exact results for comparison. Although the results obtained in this study could be evaluated as not to be in a very good accordance with the results already presented in the literature; this should be realised as these calculations were carried out using only the lowest order approximations of the polynomial expansion techniques. Then it is clear that more convenient results with the literature may be obtained in case applying higher-order aproximations. However, it is important to underline that the $U_{1}$ method was first pplied to the problem and an analytical expression for the extrapolation distance was derived explicitly.
\end{abstract}

Keywords: $\mathrm{P}_{1}$ method, $\mathrm{U}_{1}$ method, extrapolation distance

\section{$U_{1}$ Yöntemi Kullanılarak Uzatılmış Mesafe Probleminin Çözümü Üzerine Bir Çalışma}

\section{Özet}

Homojen bir dilimde tek hızlı nötronlar için nötron transport denklemi, uzatılmış (ekstrapolasyon) mesafesi probleminin çözümü için çözülmüştür. Problemin çözüm algoritmasında önce nötron açısal akısının Legendre polinomları cinsinden seriye açıldığı geleneksel $P_{1}$ yöntemi, ardından nötron açısal

\footnotetext{
${ }^{1}$ MSc.Osmaniye Korkut Ata University, Department of Physics, cevataksu@ hotmail.com.tr

${ }^{2}$ Corresponding Author; Prof.Dr., Osmaniye Korkut Ata University, Department of Physics, hakanozturk@osmaniye.edu.tr ORCID NO: 0000-0001-8136-2406
} 
akısının ikinci tür Chebyshev polinomları cinsinden seriye açıldığı $U_{1}$ yöntemi kullanılmıştır. Literatürde $P_{1}$ yöntemi ile elde edilen problemin çözümünü bulmak mümkün iken, bu probleme ilk olarak $U_{1}$ yöntemi uygulanmış ve bu çalışmanın temel amacı olarak analitik bir ifade elde edilmiş̧ir. Son olarak, her iki yöntem kullanılarak uzatılmış mesafeler için nümerik sonuçlar hesaplanmış ve karşılaştırma için tam sonuçlarla birlikte tablolarda verilmiştir. Bu çalışmada elde edilen sonuçların literatürde halihazırda sunulan sonuçlarla çok iyi bir uyum içinde olmadığı değerlendirilebilse de, bu hesaplamalar polinom açılım tekniklerinin sadece en düşük mertebeli yaklaşımları kullanılarak yapıldığından buna göre değerlendirilmesi gereklidir. $\mathrm{O}$ halde daha yüksek mertebeden yaklaşımların uygulanması durumunda literatürle daha uygun sonuçların elde edilebileceği açıktır. Ancak, probleme U1 yönteminin ilk defa uygulandığının ve uzatılmış mesafe için analitik bir ifadenin açıkça türetildiğinin altını çizmek önemlidir.

Anahtar kelimeler: $\mathrm{P}_{1}$ yöntemi, $\mathrm{U}_{1}$ yöntemi, uzatılmış mesafe

\section{Introduction}

There are many problems which are in the interests of the scientists who deal with the solution of the neutron transport equation in standard geometries. Extrapolation distance (extrapolated end point) $z_{0}$ or the linear extrapolation distance $d$ is one of those problems and this concept describes the distance at which the neutron flux is thought to be extrapolated to zero at the boundary of the system. If the neutron flux calculated from the diffusion equation is assumed to vanish at a small distance $d$ beyond the surface, then the flux determined from the diffusion equation is very nearly equal to the exact flux in the interior of the medium. The assumption that the flux vanishes a small distance $d$ beyond the surface is clearly nonphysical. Rather, it is a convenient mathematical approximation that provides a high degree of accuracy for estimates of the flux inside the medium (Lamarsh and Baratta, 2001).

Many methods have been used for the solution of this problem in neutron transport theory. Among them, the spherical harmonics $\left(P_{\mathrm{N}}\right)$ method in which the neutron angular flux is expanded in terms of the Legendre polynomials is one of the powerful methods developed for the solutions of the problems in transport theory. Therefore, the results obtained by this traditional method are accepted as a benchmark in many studies (Bell and Glasstone, 1972; Lee and Dias, 1984).

However, it is not the unique one valid for all cases. Therefore in this study, we preferred to use $U_{\mathrm{N}}$ method (Chebyshev polynomials of the second kind approximation) for the solution of the extrapolation distance for one-speed neutrons in a slab reactor. This method has been used successfully before and it can be applied to other problems of the transport theory (Conkie, 1959; Yabushita, 1961; Aspelund, 1958). In the following years, $U_{\mathrm{N}}$ method was successfully applied to problems of the transport theory (Öztürk, 2008; 2012; Öztürk and Yapar, 2018). Therefore in this study, the first order approximation ( $U_{1}$ method) in which the neutron angular flux is expanded in terms of the Chebyshev polynomials of second kind was performed for the calculation of the extrapolated end-points $z_{0}$ in a slab for various values of the $c$ (the mean number of secondary neutrons per collision). Mark and Marshak boundary conditions were used for these calculations (Lee and Dias, 1984). The numerical results obtained by $U_{1}$ and $P_{1}$ methods were tabulated in the table and they were compared with the ones available in the literature. Mathematical progresses of the methods were given in section 2 and the numerical results obtained by the methods were given in section 3. A brief conclusion was also presented in section 4.

\section{Material and Methods}

\section{$2.1 P_{1}$ approximation for extrapolation distance}


The linear transport equation in one-dimensional (slab) geometry with no source and isotropic scattering can be written as (Case and Zweifel, 1967),

$$
\mu \frac{d \psi(x, \mu)}{d x}+\sigma_{T} \psi(x, \mu)=\frac{c \sigma_{T}}{2} \int_{-1}^{1} \psi\left(x, \mu^{\prime}\right) d \mu^{\prime}, \quad-a \leq x \leq a,-1 \leq \mu \leq 1
$$

where $\mu$ is the cosine of the scattering angle with $x$-axis between the neutron velocity vectors before and after a collision. $\psi(x, \mu)$ is the neutron angular flux or neutron flux density at position $x$ traveling in direction $\mu$ and $\sigma_{T}$ is the total differential cross section.

In the first part of this study, the conventional $P_{\mathrm{N}}$ method in which the neutron angular flux $\psi(x$, $\mu$ ) is expanded in terms of the Legendre polynomials is used for the solution of the problem (Bell and Glasstone, 1972),

$$
\psi(x, \mu)=\frac{1}{2} \sum_{m=0}^{\infty}(2 m+1) P_{m}(\mu) \phi_{m}(x), \quad-a \leq x \leq a, \quad-1 \leq \mu \leq 1
$$

where $\phi_{m}(x)$ is the $m$ th Legendre moment of the angular flux and $P_{m}(\mu)$ is the Legendre polynomials; the orthogonality and recurrence relations of them which will be needed for future calculations are given by, respectively (Arfken, 1985);

$$
\begin{aligned}
& \int_{-1}^{1} P_{n}(\mu) P_{m}(\mu) d \mu= \begin{cases}2 /(2 n+1), & n=m \\
0, & n \neq m\end{cases} \\
& (2 n+1) \mu P_{n}(\mu)=(n+1) P_{n+1}(\mu)+n P_{n-1}(\mu) .
\end{aligned}
$$

Eq. (2) is inserted into Eq. (1), then the resulting equation is multiplied by $P_{m}(\mu)$ and integrated over $\mu$ in the interval $[-1,1]$ using Eqs. (3) and (4), one can obtain the $P_{N}$ moments of the angular flux as,

$$
\begin{aligned}
& \frac{d \phi_{1}(x)}{d x}+\sigma_{T} \phi_{0}(x)=c \sigma_{T} \phi_{0}(x), \\
& \frac{d \phi_{0}(x)}{d x}+2 \frac{d \phi_{2}(x)}{d x}+3 \sigma_{T} \phi_{1}(x)=0,
\end{aligned}
$$

and in general,

$$
(n+1) \frac{d \phi_{n+1}(x)}{d x}+n \frac{d \phi_{n-1}(x)}{d x}+\sigma_{T}(2 n+1) \phi_{n}(x)=\sigma_{S} \phi_{0}(x) \delta_{n 0}, \quad n=1,2, \ldots, N
$$

where $\phi_{-1}(x)=0$. Eqs. (5a) and (5b) are known as the $P_{1}$ equations for transport equation and the condition for $n=1$ stated in Eq. (5b) is equivalent to diffusion approximation or $P_{1}$ approximation in general formalism of the $P_{\mathrm{N}}$ method by setting $d \phi_{N+1}(x) / d x=0$ (Bell and Glasstone, 1972). Hence the condition for $N=1$ corresponds to diffusion approximation, a familiar equation known 
as Fick's law which is related with current $\phi_{1}(x)$ and the scalar flux $\phi_{0}(x)$ of the neutrons in the system can be obtained by setting $d \phi_{2}(x) / d x=0$ in Eq. (5b).

A general solution for Eqs. (5) is assumed as (Case and Zweifel, 1967),

$$
\phi_{n}(x)=G_{n}(v) \exp \left(-\sigma_{T} x / v\right) .
$$

When Eq. (6) was inserted into Eqs. (5), one could obtain analytical expressions for all $A_{n}(v)$ as,

$$
\begin{aligned}
& G_{1}(v)-v(1-c) G_{0}(v)=0, \\
& G_{0}(v)+2 G_{2}(v)-3 v G_{1}(v)=0,
\end{aligned}
$$

and in general,

$$
(n+1) G_{n+1}(v)+n G_{n-1}(v)+(2 n+1) v G_{n}(v)-v c G_{0}(v) \delta_{n, 0}=0, \quad n=1,2, \ldots, N
$$

where $\delta_{n, 0}$ is the kronecker delta and $v$ is a constant appearing as an eigenvalue associated with the eigenfunction $G_{n}(v)$. The discrete eigenvalues $v_{k}, k=1,2, \ldots, N+1$ can be calculated by setting $G_{N+1}(v)=0$ as the requirement of the method. Since we applied the first order approximation of the method $\left(P_{1}\right)$, we, therefore, set $G_{2}(v)=0$ in Eqs. (7) and obtained two linear equations with two unknowns; $G_{0}(v)$ and $G_{1}(v)$. This set of equations ( $7 \mathrm{a}$ and $7 \mathrm{~b}$ ) can be solved either linearly or by constructing a matrix equation. Then, this set of the equation can be solved to obtain the eigenvalues as,

$$
v_{1,2}= \pm \frac{1}{\sqrt{3(1-c)}} .
$$

In order to investigate the extrapolation distance problem, it is remembered that the linear combination of the eigenfunctions is also a solution (Davison, 1958):

$$
\phi_{n}(x)=\sum_{j} G_{j} G_{n}\left(v_{j}\right) e^{-\sigma_{T} x / v_{j}} .
$$

when Eq. (9) was put into Eq. (2) and by applying the $P_{1}$ approximation, it is obtained,

$$
\psi(x, \mu)=\frac{1}{2}\left\{G_{1}\left[1+3 \mu G_{1}\left(v_{1}\right)\right] e^{-\sigma_{T} x / v_{1}}+G_{2}\left[1+3 \mu G_{1}\left(v_{2}\right)\right] e^{-\sigma_{T} x / v_{2}}\right\}
$$

where $G_{0}\left(v_{j}\right)=1$. Eq. (7a) and Eq. (8) are used in Eq. (10), then it is obtained,

$$
\psi(x, \mu)=\frac{1}{2}\left\{G_{1}[1+\mu \sqrt{3(1-c)}] e^{-\sigma_{T} x \sqrt{3(1-c)}}+G_{2}[1-\mu \sqrt{3(1-c)}] e^{\sigma_{T} x \sqrt{3(1-c)}}\right\} .
$$


As well known, it is necessary to know some boundaries or initial conditions to solve the problems. It is also needed free surface boundary and symmetry conditions:

$$
\begin{aligned}
& \psi( \pm a, \mu)=0, \\
& \psi(x, \mu)=\psi(-x, \mu), \quad \mu>0 .
\end{aligned}
$$

Mark and Marshak boundary conditions are widely used in the solution of the problems of the neutron transport theory. Mark used the concept of continuity of the angular flux, which implies the continuity of all the angular moments of the neutron flux across the boundaries surrounded by the vacuum, and showed that is condition is equivalent to zero incoming angular flux at the boundaries for the specific values of $\mu$ (Bell and Glasstone, 1972). Then, the Mark boundary condition can be described as;

$$
\psi\left(0, \mu_{k}\right)=0, \quad(N+1) / 2<k \leq(N+1),
$$

where $\mu_{k}$ is the $k$ th negative root of $P_{N+1}(\mu)=0$. When Eq. (11) is inserted in Eq. (13), it is obtained,

$$
A_{1}[1+\mu \sqrt{3(1-c)}]+A_{2}[1-\mu \sqrt{3(1-c)}]=0 .
$$

The scalar flux density $\phi_{n}(x)$ in slab geometry can be written as,

$$
\phi_{n}(x)=\int \psi(x, \mu) P_{n}(\mu) d \Omega,
$$

where $d \Omega=\sin \theta d \theta d \varphi$ and $\mu=\cos \theta ; 0 \leq \theta \leq \pi$ and $0 \leq \varphi \leq 2 \pi$. When Eq. (15) is written for $n=0$ in which the neutron angular flux given by Eq. (11) is used, we obtained,

$$
\phi_{0}(x)=2 \pi\left\{A_{1} e^{-\sigma_{T} x \sqrt{3(1-c)}}+A_{2} e^{\sigma_{T} x \sqrt{3(1-c)}}\right\} .
$$

The extrapolated end-point is defined as the distance from a vacuum boundary at which the asymptotic flux i.e. the flux extended by its natural curvature with distance will vanish. This is the problem for a half-space $x \geq 0$ with a source infinitely far into the interior. Mathematically, $z_{0}$ is defined in plane geometry by the condition $\quad \phi_{0}\left(-z_{0}\right)=0$, where $\phi_{0}(x)$ is the asymptotic scalar flux at a distance $x$ into the medium. The extrapolated end-point $z_{0}$ for both boundary conditions can be stated as (Davison, 1958),

$$
\phi_{0}\left(-z_{0}\right)=0
$$

Therefore, the condition for extrapolated end-point given in Eq. (17) is applied to Eq. (16);

$$
\phi_{0}\left(-z_{0}\right)=2 \pi\left\{A_{1} e^{\sigma_{T} z_{0} \sqrt{3(1-c)}}+A_{2} e^{-\sigma_{T} z_{0} \sqrt{3(-c)}}\right\}=0 .
$$

Then, a new relation between $A_{1}$ and $A_{2}$ could be obtained from Eq. (18),

$$
A_{2} e^{-\sigma_{T} z_{0} \sqrt{3(1-c)}}=-A_{1} e^{\sigma_{T} z_{0} \sqrt{3(1-c)}}
$$


Eq. (14) and Eq. (19) were solved together to find an expression for the extrapolated end point $z_{0}$ for $P_{1}$ approximation,

$$
\left(z_{0}\right)_{\text {Mark }}=\frac{1}{\sigma_{T} \sqrt{3(1-c)}} \tanh ^{-1}(\sqrt{1-c}) .
$$

The same problem was also investigated using Marshak boundary condition. The Marshak boundary condition is based on the condition of zero incoming current at the vacuum boundary (Bell and Glasstone, 1972). Therefore, it is defined as,

$$
\int_{0}^{1} P_{k}(\mu) \psi(0, \mu) d \mu=0, \quad k=1,3, . . N,
$$

It is seen in Eq. (21) that the state for only $k=1$ satisfies the condition of zero incoming current. The other values of $k$ give the additional conditions necessary to solve the problem. When Eq. (11) is replaced to Eq. (21) one can get,

$$
A_{1}\left[\frac{1}{4}+\frac{1}{6} \sqrt{3(1-c)}\right]+A_{2}\left[\frac{1}{4}-\frac{1}{6} \sqrt{3(1-c)}\right]=0 .
$$

In the case of Marshak boundary condition, Eq. (19) and Eq. (22) were solved together and an analytical expression was obtained for the extrapolated end point $z_{0}$ for $P_{1}$ approximation,

$$
\left(z_{0}\right)_{\text {Marshak }}=\frac{1}{\sigma_{T} \sqrt{3(1-c)}} \tanh ^{-1}\left(2 \sqrt{\frac{1-c}{3}}\right) .
$$

\section{$2.2 U_{1}$ approximation for extrapolation distance}

In the second part of this study, the first order approximation of the Chebyshev polynomials of second kind, i.e. $U_{1}$ method was applied to the problem of the extrapolated end-point for one-speed neutrons. The same procedure carried out in the previous section was followed since Legendre and Chebyshev polynomials are in the same polynomials family, i.e. Jacobi polynomials. Therefore, their applications to the problems are in the same manner. In this method, neutron angular flux is expanded in terms of the Chebyshev polynomials of second kind which was applied successfully in previous studies (Öztürk, 2008; 2012),

$$
\psi(x, \mu)=\frac{2}{\pi} \sqrt{1-\mu^{2}} \sum_{n=0}^{N} \phi_{n}(x) U_{n}(\mu), \quad-a \leq x \leq a,-1 \leq \mu \leq 1 .
$$

In order to obtain the $U_{\mathrm{N}}$ moments of the angular flux, one should replace Eq. (24) into Eq. (1) and then multiply the resultant equation by $U_{m}(\mu)$ and integrate over $\mu$ in the interval $[-1,1]$. During these derivations, the orthogonality and the recurrence relations of the Chebyshev polynomials of second kind are needed,

$$
\int_{-1}^{1} U_{n}(\mu) U_{m}(\mu) \sqrt{1-\mu^{2}} d \mu= \begin{cases}\pi / 2, & n=m \\ 0, & n \neq m\end{cases}
$$




$$
2 \mu U_{n}(\mu)=U_{n+1}(\mu)+U_{n-1}(\mu), \quad-1 \leq \mu \leq 1 .
$$

Under these circumstances, the $U_{\mathrm{N}}$ moments of the angular flux can be obtained for $n=0$ and $n=$ 1 , respectively;

$$
\begin{array}{r}
\frac{d \phi_{1}(x)}{d x}+2 \sigma_{T}(1-c) \phi_{0}(x)=0, \\
\frac{d \phi_{2}(x)}{d x}+\frac{d \phi_{0}(x)}{d x}+2 \sigma_{T} \phi_{1}(x)=0,
\end{array}
$$

and in general,

$$
\frac{\mathrm{d} \phi_{n+1}(x)}{\mathrm{d} x}+\frac{\mathrm{d} \phi_{n-1}(x)}{\mathrm{d} x}+2 \sigma_{T} \phi_{n}(x)-c \sigma_{T}\left\{\frac{\left[1+(-1)^{n}\right]}{n+1} \phi_{0}(x)\right\}=0, \quad n \geq 1 .
$$

Eqs. (26a) and (26b) are known as the $U_{1}$ equations for the transport equation and the condition for $n=1$ stated in Eq. (26b) is equivalent to diffusion approximation or $U_{1}$ approximation in the general formalism of the $U_{\mathrm{N}}$ method by setting $d \phi_{N+1}(x) / d x=0$ (Bell and Glasstone, 1972). A general solution for Eqs. (26) is assumed as (Bell and Glasstone, 1972),

$$
\phi_{n}(x)=G_{n}(v) \exp \left(-\sigma_{T} x / v\right) .
$$

When Eq. (27) is replaced into Eqs. (26), analytical expressions for all $A_{n}(v)$ could be obtained as,

$$
\begin{aligned}
& G_{1}(v)-2 v(1-c) G_{0}(v)=0, \\
& G_{0}(v)+G_{2}(v)-2 v G_{1}(v)=0,
\end{aligned}
$$

and in general,

$$
G_{n+1}(v)+G_{n-1}(v)+2 v G_{n}(v)-v c\left[\frac{1+(-1)^{n}}{n+1}\right] G_{0}(v)=0, \quad n \geq 1 .
$$

The discrete eigenvalues $v_{k}, k=1,2, \ldots, N+1$ can be calculated by setting $G_{N+1}(v)=0$ as the requirement of the method. In the first order approximation of the method $\left(U_{1}\right), A_{2}(v)=0$ is set in Eqs. (28a) and two linear equations with two unknowns $A_{0}(v)$ and $A_{1}(v)$ are obtained. This set of equations (28a and $28 \mathrm{~b}$ ) can be solved either linearly or by constructing a matrix equation. When this set of the equation is solved, the eigenvalues can be obtained as,

$$
v_{1,2}= \pm \frac{1}{2 \sqrt{(1-c)}}
$$

By following the same procedure with the $P_{\mathrm{N}}$ method mentioned in the previous section, the solution for the eigenvectors can be written as (Davison, 1958): 


$$
\phi_{n}(x)=\sum_{j} G_{j} G_{n}\left(v_{j}\right) e^{-\sigma_{T} x / v_{j}} .
$$

When Eq. (30) was put into Eq. (24) and by applying the $U_{1}$ approximation,

$$
\psi(x, \mu)=\frac{2}{\pi} \sqrt{1-\mu^{2}}\left\{A_{1}\left[1+2 \mu A_{1}\left(v_{1}\right)\right] e^{-\sigma_{T} x / v_{1}}+A_{2}\left[1+2 \mu A_{1}\left(v_{2}\right)\right] e^{-\sigma_{T} x / v_{2}}\right\}
$$

where $A_{0}\left(v_{j}\right)=1$. An explicit form of Eq. (31) can be obtained by using Eq. (28a) and Eq. (29) in Eq. (31),

$$
\psi(x, \mu)=\frac{2}{\pi} \sqrt{1-\mu^{2}}\left\{A_{1}[1+2 \mu \sqrt{1-c}] e^{-2 \sigma_{T} x \sqrt{1-c}}+A_{2}[1-2 \mu \sqrt{1-c}] e^{2 \sigma_{T} x \sqrt{1-c}}\right\} .
$$

The transport equation should satisfy certain physical properties of the system at vacuum boundaries, that is, free surface boundary and symmetry conditions:

$$
\begin{aligned}
& \psi( \pm a, \mu)=0, \\
& \psi(x, \mu)=\psi(-x, \mu), \quad \mu>0 .
\end{aligned}
$$

As in the first part of this study, first the Mark boundary condition is applied;

$$
\psi\left(0, \mu_{k}\right)=0, \quad(N+1) / 2<k \leq(N+1),
$$

where $\mu_{k}$ is the $k$ th negative root of $U_{N+1}(\mu)=0$. When Eq. (32) is applied to the Mark boundary condition given in Eq. (34), it is obtained,

$$
A_{1}[1+\mu \sqrt{2(1-c)}]+A_{2}[1-\mu \sqrt{2(1-c)}]=0
$$

The scalar flux density $\phi_{n}(x)$ can be written similar to Eq. (15),

$$
\phi_{n}(x)=\int \psi(x, \mu) U_{n}(\mu) d \Omega,
$$

After Eq. (32) is inserted into Eq. (36), it is written for $\phi_{0}(x)$,

$$
\phi_{0}(x)=2 \pi\left\{A_{1} e^{-2 \sigma_{T} x \sqrt{1-c}}+A_{2} e^{2 \sigma_{T} x \sqrt{1-c}}\right\} .
$$

From the definition of extrapolated end-point given in Eq. (17), Eq. (37) can be rearranged as;

$$
\phi_{0}\left(-z_{0}\right)=2 \pi\left\{A_{1} e^{2 \sigma_{T} z_{0} \sqrt{1-c}}+A_{2} e^{-2 \sigma_{T} z_{0} \sqrt{1-c}}\right\}=0 \text {. }
$$

A relation between $A_{1}$ and $A_{2}$ can be written from Eq. (38) as, 


$$
A_{2} e^{-2 \sigma_{T} z_{0} \sqrt{1-c}}=-A_{1} e^{2 \sigma_{T} z_{0} \sqrt{1-c}} .
$$

At the end of the implementation of Mark boundary condition for $U_{1}$ approximation, Eq. (35) and Eq. (39) are solved together an analytical expression for the extrapolated end point $z_{0}$ can be obtained as,

$$
\left(z_{0}\right)_{\text {Mark }}=\frac{1}{2 \sigma_{T} \sqrt{1-c}} \tanh ^{-1}(\sqrt{1-c}) .
$$

In case of Marshak boundary condition,

$$
\int_{0}^{1} U_{k}(\mu) \psi(0, \mu) d \mu=0, \quad k=1,3, . . N
$$

Eq. (32) is replaced to Eq. (41), it is obtained as,

$$
A_{1}\left[\frac{4}{3 \pi}+\frac{1}{2} \sqrt{1-c}\right]+A_{2}\left[\frac{4}{3 \pi}-\frac{1}{2} \sqrt{1-c}\right]=0 .
$$

Eq. (39) and Eq. (42) are solved together and an expression for the extrapolated end point $z_{0}$ can be obtained as,

$$
\left(z_{0}\right)_{\text {Marshak }}=\frac{1}{2 \sigma_{T} \sqrt{1-c}} \tanh ^{-1}\left(\frac{3 \pi}{8} \sqrt{1-c}\right) .
$$

\section{Results and Discussion}

In this study, the extrapolation distance problem was investigated for one-speed neutrons in a slab reactor using both $P_{1}$ and $U_{1}$ approximations. The extrapolation distances were calculated for isotropic scattering for various values of the collision parameter $c$ near to unity which is reported as to be important for the calculation of diffusion approximations. Mark and Marshak boundary conditions were used for all calculations and numerical results obtained by both methods are tabulated in Table 1. In this table, the numerical results obtained from the present $U_{1}$ method together with the ones obtained from the traditional $P_{1}$ method and exact results quoted from Case et al (1953) were given side by side for comparison. In this study, this problem has been solved for the first time using $U_{1}$ approximations. All computations were carried out using Maple software and the total differential cross-section is assumed to have its normalized values, $\sigma_{T}=1 \mathrm{~cm}^{-1}$. 
Table 1: Extrapolated end points calculated for $0.00 \leq \mathrm{c} \leq 2.00$

\begin{tabular}{llllll}
\hline & \multicolumn{2}{l}{ Mark Boundary Cond. } & \multicolumn{2}{l}{ Marshak Boundary Cond. } & $\begin{array}{l}\text { Exact results } \\
\text { Case et al }\end{array}$ \\
\cline { 2 - 6 } $\boldsymbol{\boldsymbol { P } _ { \mathbf { 1 } }}$ & $\boldsymbol{U}_{\mathbf{1}}$ & $\boldsymbol{P}_{\mathbf{1}}$ & $\boldsymbol{U}_{\mathbf{1}}$ & 1.0000 \\
\hline $\mathbf{0 . 0 0}$ & - & - & - & - & 0.7207 \\
\hline $\mathbf{0 . 5 0}$ & 0.7196 & 0.6232 & 0.9359 & 0.8471 & 0.7155 \\
\hline $\mathbf{0 . 6 0}$ & 0.6805 & 0.5894 & 0.8484 & 0.7604 & 0.7127 \\
\hline $\mathbf{0 . 7 0}$ & 0.6484 & 0.5615 & 0.7858 & 0.7003 & 0.7113 \\
\hline $\mathbf{0 . 8 0}$ & 0.6212 & 0.5380 & 0.7377 & 0.6549 & 0.7106 \\
\hline $\mathbf{0 . 9 0}$ & 0.5978 & 0.5177 & 0.6989 & 0.6188 & 0.7104 \\
\hline $\mathbf{0 . 9 8}$ & 0.5812 & 0.5034 & 0.6727 & 0.5946 & 0.7104 \\
\hline $\mathbf{0 . 9 9}$ & 0.5793 & 0.5017 & 0.6697 & 0.5918 & 0.7104 \\
\hline $\mathbf{1 . 0 1}$ & 0.5754 & 0.4983 & 0.6637 & 0.5863 & 0.7106 \\
\hline $\mathbf{1 . 1 0}$ & 0.5592 & 0.4843 & 0.6392 & 0.5639 & 0.7109 \\
\hline $\mathbf{1 . 2 0}$ & 0.5429 & 0.4702 & 0.6154 & 0.5421 & 0.7113 \\
\hline $\mathbf{1 . 3 0}$ & 0.5282 & 0.4574 & 0.5954 & 0.5231 & 0.7118 \\
\hline $\mathbf{1 . 4 0}$ & 0.5148 & 0.4458 & 0.5758 & 0.5062 & 0.7177 \\
\hline $\mathbf{2 . 0 0}$ & 0.4535 & 0.3927 & 0.4948 & 0.4335 & \\
\hline
\end{tabular}

\section{Conclusion}

Extrapolated end-point problem for one-speed neutrons in a finite slab with isotropic scattering was investigated using traditional $P_{1}$ approximation and $U_{1}$ approximation. The numerical results given in Table 1 obtained for the extrapolation distance using $U_{1}$ approximation approaches to the exact results better in the case of Marshak than Mark boundary conditions, especially in case $c$ is very near to unity. Although the numerical results obtained by the $P_{1}$ approximation look etter than the results obtained by $U_{1}$ approximation, this does not mean that the present $U_{\mathrm{N}}$ method could not be applied to the problems of transport theory. Very good results are not expected in the first order approximations anyway. We hope to eliminate this handicap by applying higher order approximations in our future works. In addition, it is very important to eliminate an analytical expression firstly by applying the $U_{\mathrm{N}}$ method to this problem. This inference could lead to new applications. Finally, we indicated that deriving an analytical expression for the solution of the problem given in Eqs. (40) and (43) is very important for future studies.

\section{References}

Anli, F., Yasa, F., Güngör, S., Öztürk, H. (2006). "T ${ }_{\mathrm{N}}$ approximation to reflected slab and computation of the critical half thickness". J. Quant. Spectrosc. Radiat. Transfer 101, 135

Arfken, G. (1985). Mathematical methods for physicists. London: Academic Press, Inc. 
Aspelund, O. (1958). "On a new method for solving the (Boltzmann) equation in neutron transport theory”. PICG 16, 530

Bell, G.I., Glasstone, S. (1972). Nuclear reactor theory, New York: VNR Company

Case, K.M., de Hoffmann, F., Placzek, G. (1953). Introduction to the theory of neutron diffusion, USA: Las Alamos Scientific Laboratory

Case, K.M., Zweifel, P.F. (1967). Linear transport theory. Addison-Wesley Publishing Company

Conkie, W.R. (1959). "Polynomial approximations in neutron transport theory". Nucl. Sci. Eng., 6,260

Davison, B. (1958). Neutron transport theory. London: Oxford University Press

Lamarsh, J.R., Baratta, A.J. (2001). Introduction to Nuclear Engineering, 3rd edn. Prentice Hall Inc., Upper Saddle River

Lee, C.E., Dias, M.P. (1984). "Analytical solutions to the moment transport equations-I; one-group one-region slab and sphere criticality". Ann. Nucl. Energy 11, 515-530.

Öztürk, H. (2008). "The reflected critical slab problem for one-speed neutrons with strongly anisotropic scattering". Kerntechnik 73, 66-74

Öztürk, H. (2012). "The criticality calculations for one-speed neutrons in a reflected slab with anisotropic scattering using the modified UN method". Kerntechnik 77, 453-457

Öztürk, H., Şimşek Yapar, A. (2018). "Alternative scattering kernels for the first estimates of a reactor: diffusion length". Nucl. Sci. Tech. 29:37

Yabushita, S. (1961). "Tschebyscheff polynomials approximation method of the neutron transport equation”. J. Math. Phys. 2, 543 